\title{
The nigrostriatal dopaminergic pathway in Wilson's disease studied with positron emission tomography
}

\author{
Barry J Snow, Mohit Bhatt, W R Wayne Martin, David Li, Donald B Calne
}

\begin{abstract}
Movement disorders, including Parkinsonism, are prominent features of neurological Wilson's disease (WD). This suggests there may be dysfunction of the nigrostriatal dopaminergic pathway. To explore this possibility, five patients were studied using positron emission tomography (PET) with ${ }^{18}$ F-6-fluorodopa (6FD), and magnetic resonance imaging (MRI). We calculated striatal 6FD uptake rate constants by a graphical method and compared the results with those of 18 normal subjects. It was found that four patients with symptoms all had abnormally low 6FD uptake, and the one asymptomatic patient had normal uptake. PET evidence for nigrostriatal dopaminergic dysfunction was present even after many years of penicillamine treatment. It is concluded that the nigrostriatal dopaminergic pathway is involved in neurological WD.
\end{abstract}

Wilson's disease (WD) is an autosomal recessive disorder characterised by widespread copper deposition throughout the body, particularly in the liver and brain. ${ }^{1}$ Neurological presentation varies with combinations of psychiatric disturbance, involuntary movements, incoordination and dysarthria; pyramidal tract abnormalities are minimal, and sensation is spared. Pathological studies, ${ }^{23}$ computerised tomography $(\mathrm{CT})^{45}$ and magnetic resonance imaging (MRI) ${ }^{6-8}$ show that the predominant lesions are in the basal ganglia, particularly the lentiform nucleus.

The prominence of movement disorders, including Parkinsonism, raises the question whether there is also a predilection for the nigrostriatal dopaminergic pathway to be involved in WD. There is only limited necropsy information suggesting that the pathway may be damaged in WD. ${ }^{910}$ Although the structural integrity of the striatum and substantia nigra may be studied with CT and MRI, these modalities cannot study the functional integrity of the dopaminergic system. Until now, there has been no satisfactory method of studying the function of the nigrostriatal dopaminergic pathway in living subjects.

With the advent of positron emission tomography (PET) using the tracer ${ }^{18} \mathrm{~F}-6-$ fluorodopa (6FD), we can investigate the function of the nigrostriatal dopaminergic pathway in vivo. ${ }^{11}$ Following administration, radioactive metabolites of $6 \mathrm{FD}$ accumulate in the striatal endings of nigrostriatal dopaminergic neurons. PET can image this accumulation and thereby display the anatomical distribution of the intact nerve endings. ${ }^{12}$ In addition, tracer-uptake data gathered during the scan, can be used to derive a $6 \mathrm{FD}$ uptake rate constant. This constant is an index of the activity of the enzyme dopa-decarboxylase that metabolises dopa to dopamine. ${ }^{11}$ Necropsy studies show that dopa-decarboxylase activity decreases with age and is diminished in Parkinson's disease. ${ }^{1314}$ PET studies agree with this necropsy data, showing decreases in 6FD accumulation with age and in Parkinson's disease. ${ }^{11}{ }^{15-17}$ In addition, PET is sufficiently sensitive to demonstrate asymptomatic dopaminergic lesions in humans and monkeys. ${ }^{1819}$

WD has not previously been studied with 6FD PET. We employed this technique to determine if the nigrostriatal dopaminergic pathway is damaged in WD. We compared five patients with 18 controls.

\section{Patients}

The diagnosis of WD was based on the following established criteria in all patients: medical history, physical examination, Kayser-Fleischer rings confirmed on slitlamp examination, low serum ceruloplasmin levels and elevated urinary copper excretion (table 1 ). ${ }^{20}$ The relevant clinical features of the patients are presented below. No patient had symptomatic liver disease or had other significant medical illness. Standard liver function tests were normal in all patients at the time of scanning (alkaline phosphatase, aspartate aminotransferase, gamma-glutamyl transferase and bilirubin). None had a history of exposure to phenothiazines or other dopamine receptor antagonists. When studied, no patient had ataxia, pyramidal tract signs, or sensory changes. The control group consisted of 18 subjects (mean age $40 \cdot 2$ years, range 22-54); all were neurologically normal and none were taking medication at the time of scanning.

Patient 1 was a 23 year old man who was diagnosed as having WD at age 12 when he developed predominantly Parkinsonian features with slurred speech, clumsy hand movements, micrographia, and a slowed gait. There was an intention tremor of the right hand. He was started on penicillamine $2 \mathrm{gm}$ daily and improved steadily. When studied 
Table 1 Biochemical findings

\begin{tabular}{lll}
\hline Patient & $\begin{array}{l}\text { Ceruloplasmin } \\
(\mathrm{mg} / 100 \mathrm{ml})\end{array}$ & $\begin{array}{l}\text { Urinary Copper } \\
\text { (ug/24 hours) }\end{array}$ \\
\hline 1 & $<7$ & 930 \\
2 & $<7$ & $114^{\star}$ \\
3 & 8 & $145^{\star}$ \\
4 & 12 & $290^{\star}$ \\
5 & $<7$ & $210^{\star}$ \\
\hline
\end{tabular}

^Measurement taken while on treatment.

the only abnormal findings were mild dysarthria and impaired rapid alternating movements of the hands.

Patient 2 was a 28 year old woman who presented at age 13 with tremor of the hands, drooling and slurred speech. Her twin brother had been diagnosed as having WD two years previously. She took penicillamine for two years then stopped for unknown reasons. At age 18 she was admitted to a psychiatric ward with disinhibited behaviour, drooling and dystonic hand movements; she improved on penicillamine. At age 26 she stopped her penicillamine again and within a month developed inappropriate laughter, unintelligible speech and dystonic hand movements. On penicillamine 2 gm per day she improved. When studied she had mild dementia and disinhibition, dysarthric but intelligible speech, and dystonic posturing of the upper limbs. There was no tremor, and no Parkinsonian features.

Patient 3 was a 37 year old man who was diagnosed as having WD at age 30 when he sought an ophthalmological opinion regarding what were diagnosed as Kayser-Fleischer rings. Neurological examination revealed only a mild intention tremor of the right hand. On penicillamine $1 \mathrm{gm}$ per day the rings and tremor resolved. When studied he was neurologically normal.

Patient 4 was a 50 year old man who was diagnosed as having WD at age 18 when he developed slurring of speech, slow movements and drooling. An older brother had died of WD. Treatment with 2-3-dimercapto-pranol (BAL) resulted in a modest initial improvement, but he subsequently deteriorated. By age 30 he was bedbound with unintelligible speech, rigidity and weight loss. On penicillamine $2 \mathrm{gm}$ per day he improved considerably. When studied he had predominantly Parkinsonian features with normal mentation, masked facies, dysarthric but intelligible speech, rigidity of all limbs, clumsy rapid alternating movements, an intention tremor and a shuffling unsteady gait.

Patient 5 was a 51 year old woman who was diagnosed as having WD at age 27 when she presented with slurred speech, clumsy hands and tremor of the arms. On penicillamine $1 \mathrm{gm}$ per day her symptoms stabilised. When studied she had predominantly Parkinsonian features with mild dementia, dysarthric but intelligible speech, a resting tremor of the right hand, rigidity, clumsy rapid alternating movements and an unstable gait.

\section{Methods}

The methods employed for PET have been described in detail elsewhere. ${ }^{11}$ The UBC/ TRIUMF PETT VI system was operated in the high resolution mode. ${ }^{21}$ The system simultaneously collects data from seven contiguous axial slices with a centre to centre separation of $14.4 \mathrm{~mm}$, in-plane resolution averaging $9.2 \mathrm{~mm}$ full width half maximum (FWHM), and an average axial resolution of $11 \mathrm{~mm}$ FWHM. A transmission scan, using a ring source containing ${ }^{68} \mathrm{Ge}$, was performed before the emission scans to permit a measured attenuation correction to be applied to the emission data.

The patient's medications were withheld from the night before scanning. All subjects received $100 \mathrm{mg}$ of carbidopa one hour before the scan. $6 \mathrm{FD}(2.0-3.5 \mathrm{mCi})$ was prepared as described previously ${ }^{22}$ and administered intravenously at the start of scanning. During the scanning period 29 sequential blood samples were drawn from an indwelling radial artery catheter, and the total radioactivity in each was determined in a well counter. Twelve sequential emission scans were performed, each of 10 minutes duration.

A graphical method was employed to calculate the steady state $6 \mathrm{FD}$ uptake rate constant for the whole striatum. ${ }^{112425}$ This method incorporates both the measured blood radioactivity and the corrected striatal radioactivity. To determine striatal radioactivity, elliptical regions of interest (ROI) were applied visually on all slices in which radioactivity in excess of background was evident. To ensure inclusion of the whole striatum, a $12 \mathrm{~cm}^{2}$ ROI was used to cover the entire area of the striatum plus some of the surrounding brain. To correct for the activity from the surrounding brain and for striatal activity emanating from nonfluorodopamine metabolites of $6 \mathrm{FD}$, a ROI was applied over each posterior temporoparietal cortex and the activity recorded from this ROI was substracted from that of the striatal ROI. ${ }^{11}$

Our method of total striatal sampling with a large ROI minimises the partial volume effect that may occur in the presence of striatal atrophy. Because of the partial volume effect, PET may underestimate the radioactivity concentration in small structures as the counts are distributed over a larger area than the original structure. However, despite the wide distribution, the counts are conserved ${ }^{26}$ so a relatively large ROI will measure total radioactivity in the structure of interest. ${ }^{11}$

The graphical method requires correction for the decreasing availability of 6FD to the striatum as it is metabolised in the periphery. In particular, allowance must be made for the action of catechol-O-methyl transferase on 6FD to form 3-O-methyl-6FD (3-OMFD). We measured the plasma concentration of 3OMFD by the method described by Boyes $e t$ al and calculated the ratio of 3-OMFD to 6FD in each blood sample. ${ }^{27}$ In previous studies, we expressed the increase of this ratio with time as the slope of a straight line calculated by regression analysis. ${ }^{11}$ However, inspection of the data 
Figure 1 FD uptake constants plotted against age for five patients with Wilson's disease and 18 normal subjects. The regression line is for the normal subjects $r=0.5$ $p<0.05)$; the broken lines mark $90 \%$ confidence limits for prediction of individual values.

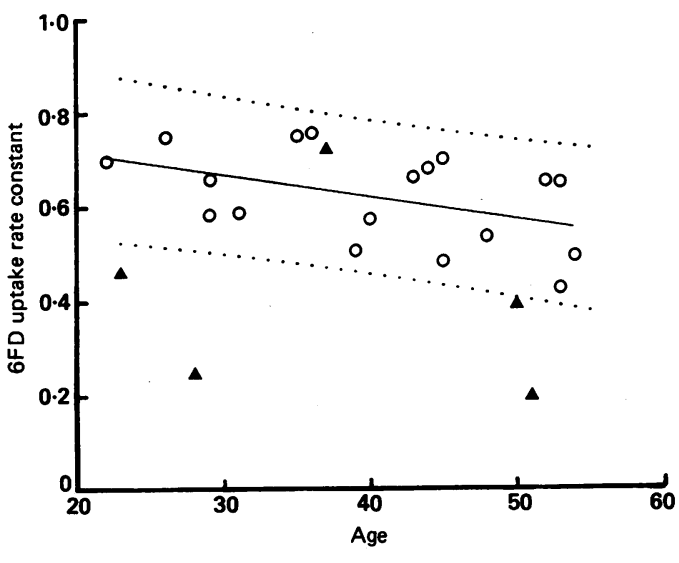

reveals that this increase is not necessarily linear. We have improved upon this method by calculating the proportion of total blood radioactivity due to $6 \mathrm{FD}$ at a series of times after tracer administration. We used these proportions to correct the blood time-activity curve and thus determine the true input function.

MRI was performed on a Picker International Cryogenic MR2000 operating at 0.15 Tesla. Twelve contiguous $1 \mathrm{~cm}$ thick slices were obtained using a double-echo spin echo (SE) sequence with repetition times (TR) of $2045 \mathrm{~ms}$ and echo delay times (TE) of 40 and $120 \mathrm{~ms}$, and an inversion recovery (IR) sequence with a TR of $2450 \mathrm{~ms}$, inversion time (TI) of $400 \mathrm{~ms}$, and TE of $40 \mathrm{~ms}$.

This study was approved by the UBC ethics committee, and informed consent was obtained from all participants.

\section{Results}

Because of the influence of age on the 6FD uptake rate constant, we plotted the constants against age to compare the patients with the control group (fig 1). Limits for prediction of

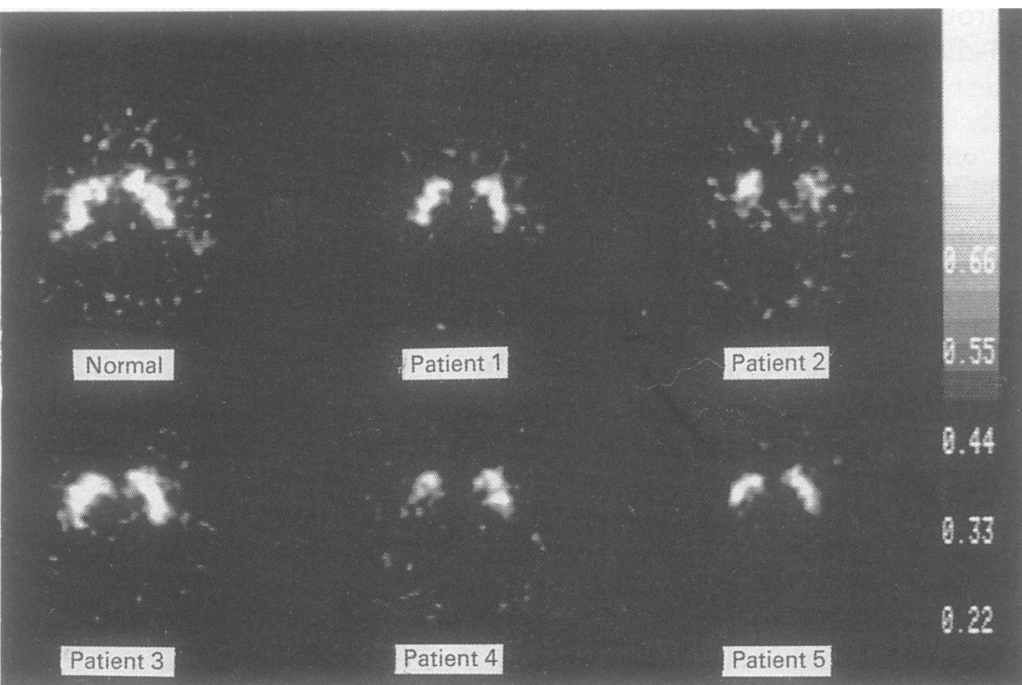

Figure 2 6FD PET images in five patients with Wilson's disease and a normal subject (age 40 years) for comparison. Figures on the scale are radioactivity concentration in arbitrary units. The images represent 30 minutes of emission data acquired during the second hour after administration of the tracer. individual values were placed at $90 \%$ to test our hypothesis of a low $6 \mathrm{FD}$ uptake rate constant at a probability of 0.05 (one-sided). Patient 3 had a normal $6 \mathrm{FD}$ uptake rate constant; patients 1 and 4 had constants just below the confidence limit of the control group; the constants of patients 2 and 5 fell well below the confidence limit of the control group.

Analysis of covariance revealed a significant difference between the normal and WD groups $(\mathrm{p}=0.001)$, with homogeneity of the slopes with age $(\mathrm{p}=0.88) .^{28}$

Selected PET and MRI images that best illustrate the abnormalities seen in each patient are shown in figs 2 and 3. The distribution of abnormal findings on MRI was similar to those reported in previous studies (table 2 ).$^{6-8}$

\section{Discussion}

We have shown that the nigrostriatal dopaminergic pathway is variably damaged in WD. The variation in 6FD uptake rate constants between our patients is consistent with the clinical, radiological and MRI heterogeneity of the disease. ${ }^{29}$ Our results reinforce the concept that WD is a multifocal process.

Previous studies have suggested that a nigrostriatal dopaminergic lesion may be present in WD. Necropsy analyses in two patients found reduced striatal dopamine and tyrosine hydroxylase. ${ }^{910}$ Another study detected reduced cerebrospinal fluid dopamine metabolites in one patient with WD, while a sibling with asymptomatic disease had normal concentrations. ${ }^{30}$ Case reports have described a range of responses to levodopa in therapeutic trials. ${ }^{3132}$ The variation may derive from different methods of study, or from different degrees of dopaminergic deficit. PET provides a more direct assessment of in vivo dopamine synthesis and storage than previous methods have allowed.

The PET images of our two patients with considerably reduced 6FD uptake suggest that the main loss of striatal radioactivity accumulation is in the region of the putamen with relative sparing of the caudate (patients 2 and 5 , fig 2). This finding is consistent with necropsy observations suggesting preferential involvement of the putamen in WD. ${ }^{3}$

Despite the normal routine liver function tests, it is theoretically possible that hepatic dysfunction associated with WD may have increased plasma amino acid-levels which may in turn have competed with 6FD for transport across the blood-brain barrier. We investigated this by comparing the background activity (corrected for the dose of radioactivity injected) of the WD patients with the background activity of the control subjects. There was no significant difference [WD $0.22(0.052)$, controls $0.21(0.056)$, arbitrary units of radioactivity within the posterior temporo-parietal cortex ROIs]. Previous studies of 6FD PET with simultaneous amino-acid infusions in humans $\mathrm{s}^{33}$ and monkeys (unpublished observations) have shown a decrease in background radioactivity. Thus we conclude that the decrease in striatal 6FD uptake seen in our 


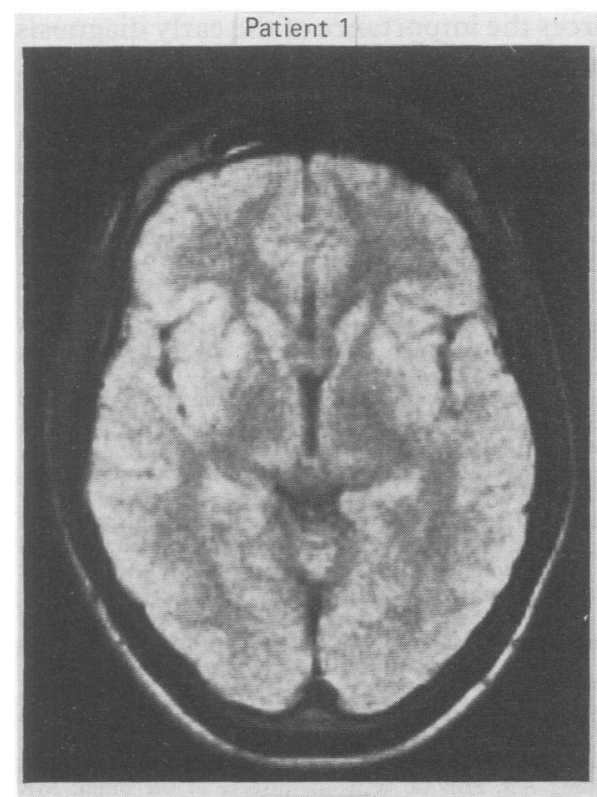

Patient 4

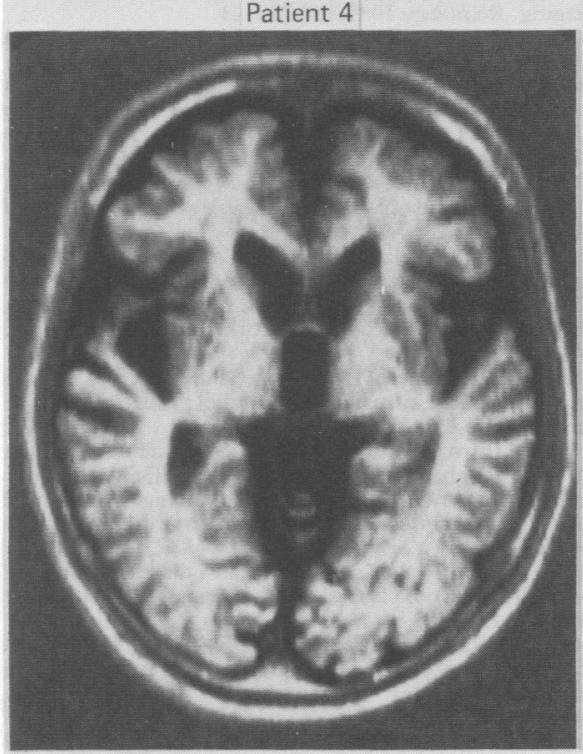

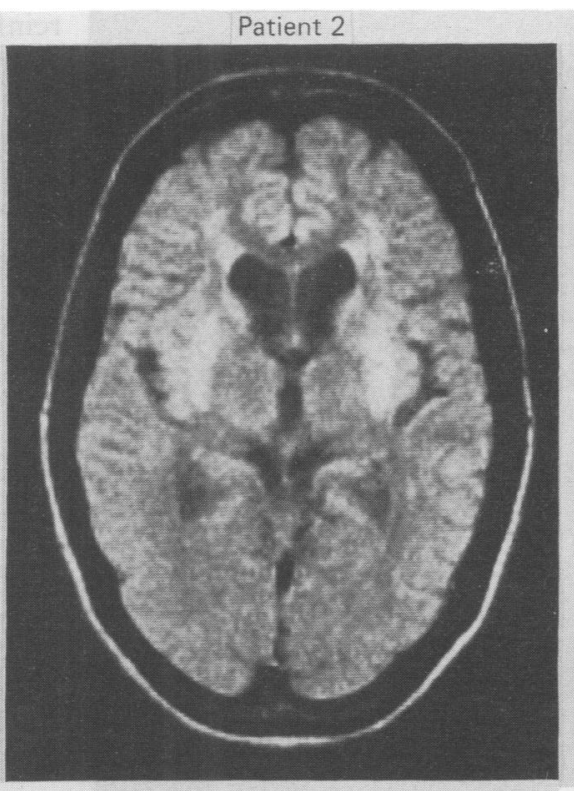

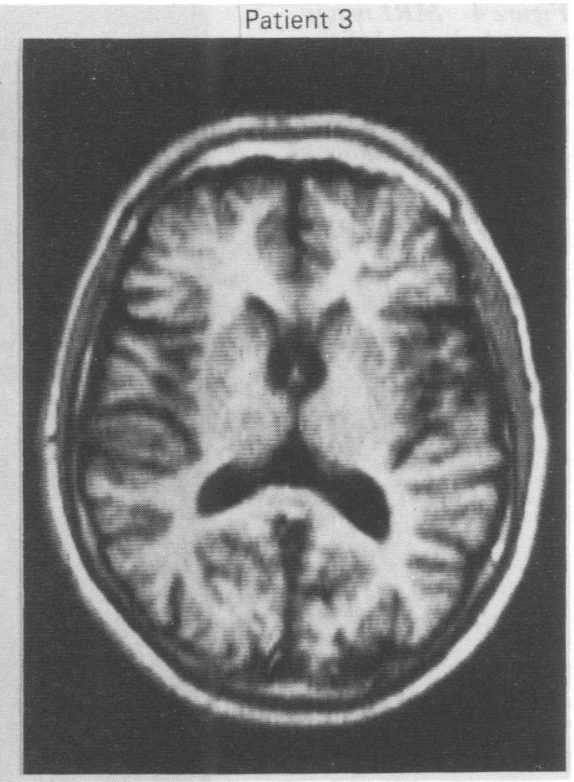

Patient 5

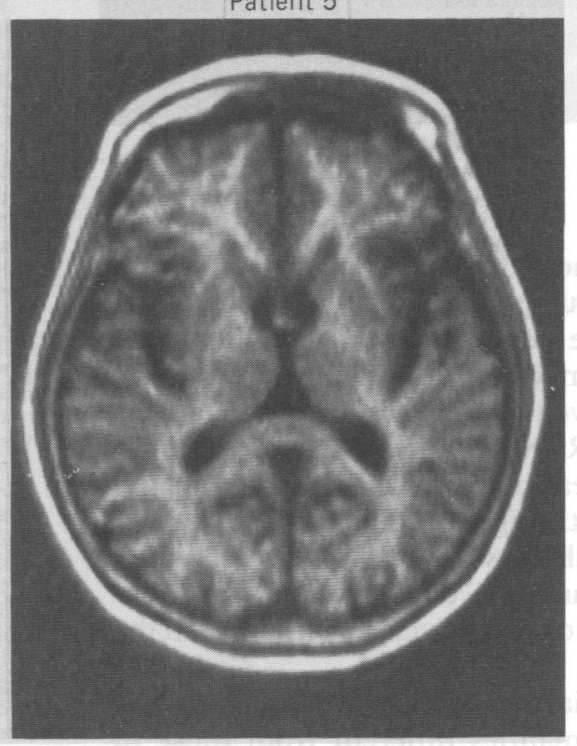

Table 2 MRI Findings

patients did not derive from competition at the blood-brain barrier.

Among the patients with abnormal PET, there were quantitative differences in the 6FD uptake rate constants that may explain some of the clinical features of WD. Clinically, patients 1, 4 and 5 had predominantly Parkinsonian features. On PET, patients 1 and 4 had mildly abnormal 6FD uptake rate constants, and on MRI both had mild striatal abnormalities. Thus their Parkinsonism may have derived from a combination of damage to dopaminergic nigrostriatal pathway neurons and damage to non-dopaminergic neurons intrinsic to the striatum. ${ }^{34}$ In contrast, patient 5 had a markedly reduced $6 \mathrm{FD}$ uptake rate constant without MRI abnormalities of the basal ganglia. The lesion that produced her Parkinsonism was probably confined to the nigrostriatal dopaminergic pathway.

The findings in patient 5 also show that there may be dysfunction of the dopaminergic pathway in WD without lesions detected by MRI. This is in accord with PET studies of WD that demonstrate decreased glucose metabolism preceding radiological changes. ${ }^{35}$ We do not

\begin{tabular}{llll}
\hline Patient & Striata & Midbrain & Cerebral Hemispheres \\
\hline 1 & Increased signal from putamen & Atrophy & N \\
2 & Caudate atrophy & Atrophy & Mild atrophy \\
3 & Increased signal from caudate and putamen & Increased signal from the colliculi & Increased signal from periventricular white matter \\
4 & $\mathrm{~N}$ & Atrophy & Mild atrophy \\
5 & $\mathrm{~N}$ & Atrophy & Atrophy \\
\end{tabular}

No patient had focal MRI abnormalities in the region of the substantia nigra. $\mathrm{N}=$ no abnormality in the structure. 
Figure 4 MRI image through the level of the midbrain of Patient 2 showing the midbrain atrophy and the increased signal from the colliculi (spin echo.).

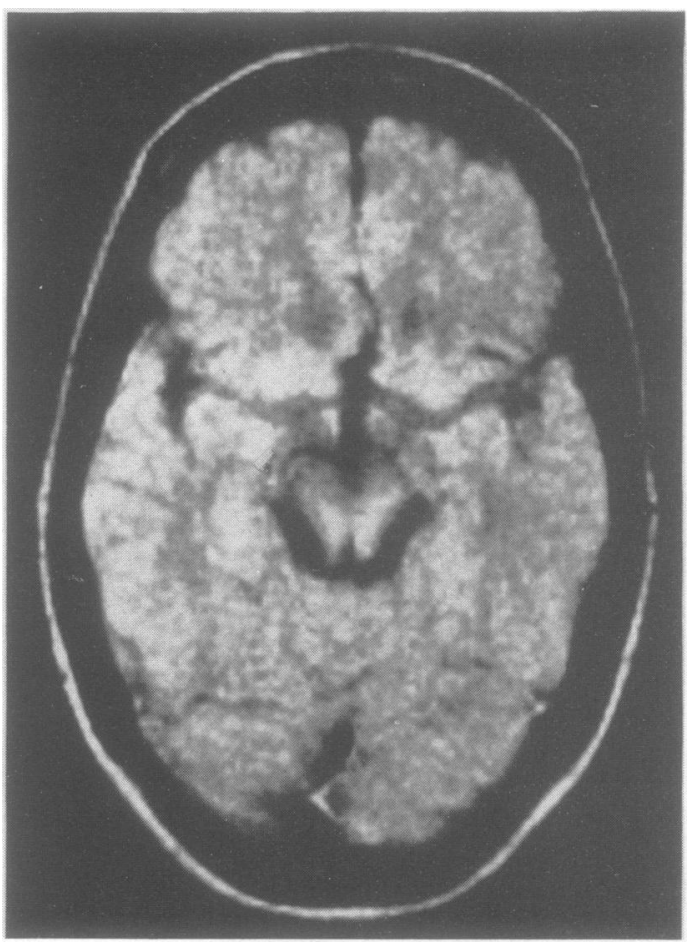

reinforces the importance of the early diagnosis and prompt treatment of WD.

We thank Sandra Cooper for her assistance with scanning as well as the members of the UBC/TRIUMF PET team. Dr Michael Schulzer assisted with statistical analysis. This study was supported by the Dystonia Medical Research Foundation, the American, the Zealand Neurological Foundation.

1 Cummings JN. The copper and iron content of brain and liver in the normal and in hepato-lenticular degeneration. liver in the normal and
Brain 1948;71:410-41.

2 Wilson SAK. Progressive lenticular degeneration: A familial nervous disease associated with cirrhosis of the liver. Brain 1912;34:295-507.

3 Scheinberg IH, Sternleib I. Wilson's disease. Philadelphia, WB Saunders, 1984.

4 Williams FJB, Walshe JM. Wilson's disease: an analysis of the cranial computerised tomographic appearances found in 60 patients and the changes in response to treatment with chelating agents. Brain 1981;104:735-54.

5 Dettori P, Rachele MG, Demelia L, et al. Computerised cranial tomography in presymptomatic and hepatic form of Wilson's disease. European Neurology 1984;23:56-63.

6 Lawler GA, Pennock JM, Steiner RE, Jenkins WJ, Sherlock S, Young IR. Nuclear magnetic resonance in Wherlock S, Young IR. Nuclear magnetic resonance

7 Aisen AM, Martel W, Gabrielsen TO, Glazier GM, Young AB, Hill G. Wilson's disease of the brain: MR imaging. Radiology 1985;157:137-41.

8 Starosta-Rubenstein S, Young AB, Kluin K, Hill G, Aisen AM, Gabrielson T, Brewer GJ. Clinical assessment of 31 patients with Wilson's disease: correlations with structural changes on magnetic resonance imaging. Arch Neurol 1987;44:365-70.

9 Nyberg P, Gottfries CG, Holmgren G, Persson S, Roos BE, Winblad B. Advanced catecholaminergic disturbances in the brain in a case of Wilson's disease. Acta Neurol Scand the brain in a case

know if the dopaminergic dysfunction observed in our patients resulted from direct damage to the nerve bodies in the substantia nigra, or from damage to the striatal nerve endings followed by retrograde death of axons. Although MRI with a higher field strength may have revealed abnormalities in the region of the substantia nigra in some of our patients, ${ }^{8}$ such abnormalities would have neither confirmed nor quantified dopaminergic dysfunction-this can only be revealed with PET in the living subject.

The dystonia of patient 2 was associated with striatal abnormalities different from those of the Parkinsonian patients. Despite the marked reduction in her $6 \mathrm{FD}$ uptake rate constant, she had no clinical Parkinsonism. On MRI there was atrophy of the caudate and SE abnormalities of the putamen that were much more severe than in patients 1 and 4 . These findings are consistent with the proposal that selective loss of dopaminergic input to a relatively intact striatum results in Parkinsonism, while destruction of the putamen may cause dystonia. ${ }^{36} 37$ J $M$ Walshe has suggested this pattern of disturbance in WD on pathological grounds. ${ }^{29}$ The common experience that patients with the dystonic form of WD are less likely to respond to chelating therapy is consistent with the notion that these patients have more extensive striatal damage.

In summary, we have shown that nigrostriatal dopaminergic dysfunction is a feature of neurological WD. This dysfunction may occur without focal abnormalities on low-field MRI and may remain after many years of penicillamine treatment. In addition, previously undetected lesions of the dopaminergic system may explain some of the differences in the neurological presentation of patients. The presence and persistence of these lesions
10 Nagatsu T, Kato T, Nagatsu I, et al. Catecholamine-related enzymes in the brain of patients with parkinsonism and Wilson's disease. In: Poirer LJ, Sourkes TL, Bedard PJ, Wilson's disease. In: Poirer LJ, Sourkes TL, Bedard PJ,
eds. Adv Neurol vol 24. New York: Raven Press, 1979: eds. $A d v$

11 Martin WRW, Palmer MR, Patlak CS, Calne DB. Nigrostriatal function in man studied with positron emission tomography. Ann Neurol 1989;26:535-42.

12 Garnett ES, Firnau G, Nahimas C. Dopamine visualised in the basal ganglia of living man. Nature 1983;305:137-8.

13 Cote LJ, Kremzner LT. Biochemical changes in normal aging in human brain. In: Mayeux R, Rosen WG, eds. The Dementias. New York: Raven Press, 1983:19-30.

14 Lloyd KG, Hornykiewicz O. Parkinson's disease: activity of L-DOPA decarboxylase in discrete brain regions. Science 1970;170:1212-13.

15 Martin WRW, Palmer MR, Peppard RF, Calne DB. Quantification of presynaptic dopaminergic function with 39(suppl): 163 .

16 Nahmias C, Garnett ES, Firnau G, Lang A. Striatal dopamine distribution in parkinsonian patients during life. J Neurol Sci 1985;69:223-30.

17 Martin WRW, Stossel AJ, Adam MJ, et al. Positron emission tomography in Parkinson's disease: glucose and dopa metabolism. In: Yahr MD, Bergman KJ, eds. Parkinson's disease. Advances in Neurology, Vol 45. New York: Raven Press; 1986:95-8.

18 Calne DB, Langston JW, Martin WRW, et al. Positron emission tomography after MPTP: observations relating emission tomography after MPTP: observations relating
to the cause of Parkinson's disease. Nature 1985;317: to the $246-8$.

19 Guttman M, Yong VW, Kim SU, Calne DB, Martin WRW, Adam MJ, Ruth TJ. Asymptomatic striatal dopamine Synapse 1988;2:469-73. Marsden CD, Fahn S, eds. Movement Disorders 2. London: Butterworths, 1987:288-302.

21 Evans B, Harrop R, Heywood D, et al. Engineering developments on the UBC-TRIUMF modified PETT VI positron emission tomograph. IEEE Trans Nucl Sci 1983;NS30:707-10.

22 Ruth TJ. The production of ${ }^{18} \mathrm{~F}-\mathrm{F}_{2}$ and ${ }^{15} \mathrm{O}-\mathrm{O}_{2}$ sequentially from the same target chamber. Int J Appl Radiat Isot 1985;36:107-10.

23 Adam MJ, Jivan S. Synthesis and purification of L- $\left[{ }^{18} \mathrm{~F}\right] 6-$ fluorodopa. Int $J$ Appl Radiat Isot 1988;39:1203-6.

24 Patlak CS, Blasberg RG, Fenstermacher JD. Graphical evaluation of blood-to-brain transfer constants from mul-
tiple-time uptake data. J Cereb Blood Flow Metabol tiple-time upt

25 Patlak CS, Blasberg RG. Graphical evaluation of blood-tobrain transfer constants from multiple-time uptake data: generalisations. J Cereb Blood Flow Metabol 1985;5: 584-90.

26 Hoffman EJ, Phelps ME. Positron emission tomography: principles and quantitation. In: Phelps ME, Mazziotta JC, Schelbert HR, eds. Positron emission tomography and autoradiography. New York: Raven Press, 1986:237-86.

27 Boyes BE, Cumming P, Martin WRW, McGeer EG. Determination of plasma $\left[{ }^{18} \mathrm{~F}\right]$-6-fluorodopa during positron emission tomography. Neurology 1989; depletion: PET scans in unilateral MPTP monkeys.

20 Sternlieb I, Giblin DR, Scheinberg H. Wilson's disease. In: 
positron emission tomography: elimination and metabolism in carbidopa treated subjects. Life Sci 1986;39.

2243-52.

28 Armitage P, Berry G. Statistical methods in medical research. Oxford: Blackwell Scientific, 1987.

29 Walshe JM. Wilson's disease: yesterday, today, and tomorrow. Movement Dis 1988;3:10-29.

30 Nijeholt JL, Korf J. Wilson's disease and monoamines. Arch Neurol 1978;35:617.

31 Barbeau A, Friesen $H$. Treatment of Wilson's disease with L-dopa after failure with penicillamine. Lancet 1970;i:1180-1.

32 Morgan JP, Preziosi TJ, Bianchine JR. Ineffectiveness of Ldopa as supplement to penicillamine in a case of Wilson's disease. Lancet 1970;ii:659.

33 Leenders KL, Poewe WH, Palmer AJ, Brenton DP,
Frackowiak RSJ. Inhibition of $\mathrm{L}-\left[{ }^{18} \mathrm{~F}\right]$ fluorodopa uptake into human brain by amino acids demonstrated by positron emission tomography. Ann Neurol 1986;20: 258-62.

34 Albin RL, Young AB, Penney JB. The functional anatomy of basal ganglia disorders. TINS 1989;12:366-75.

35 Hawkins RA, Mazziotta JC, Phelps ME. Wilson's disease studied with FDG and positron emission tomography. Neurology 1987;37:1707-11.

36 Calne DB, Martin WRW. Chemistry of the basal ganglia. In: Vinken PJ, Bruyn GW, Klawans HL, eds. Handbook of Clinical Neurology, Extrapyramidal Disorders. Amsterdam: Elsevier, 1986:33-46.

37 Fross RD, Martin WRW, Li D, et al. Lesions of the putamen: their relevance to dystonia. Neurology 1987;37:1125-9. 